\title{
Verification of Radiation Pattern Control Using a Cylindrical Metasurface
}

\author{
Brian O. Raeker and Scott M. Rudolph \\ US Naval Research Laboratory \\ Washington DC, USA
}

\begin{abstract}
We present transmission measurement results of a cylindrical impedance metasurface used to reshape the radiation pattern of an enclosed two-dimensional current line source. The metasurface was fabricated using printed circuit board methods and measured in a parallel plate waveguide. Measurement results are in agreement with the desired azimuthal image pattern, validating the design procedure of the cylindrical metasurface.
\end{abstract}

\section{INTRODUCTION}

Electromagnetic metamaterials have been demonstrated to exhibit extreme control over propagating electromagnetic waves and extensively used to influence the behavior of plane waves. Correspondingly, many metamaterial designs are implemented in planar form to suit plane wave manipulation [1]-[3], but this limits performance if cylindrical wavefronts are to be used. Additionally, while planar metamaterial designs successfully influence radiation pattern characteristics over a small angular range, they are not well-suited for producing a desired azimuthal pattern.

In a previous article, we proposed an analytical design procedure for a cylindrical impedance metasurface that interacts with cylindrical wave fronts to alter the azimuthal electric field pattern of a source [4]. An example design was presented, which introduced an azimuthal step function into the radiation pattern of a current line source. The example metasurface was designed to be implemented using printed circuit board (PCB) methods in a similar manner to the near-field plate design in [5]. Here, we detail the fabrication of the PCB metasurface and present transmission measurement results.

\section{Metasurface Design}

The cylindrical metasurface was designed to introduce an azimuthal step function into the isotropic radiation pattern of a current line source. This action is achieved by providing the necessary electric field boundary conditions between the radiation pattern emitted by the source and the desired radiation pattern at the metasurface boundary. These boundary conditions are implemented through a surface impedance distribution, calculated in [4]. This discrete impedance distribution, which includes resistance in addition to reactance, was implemented with PCB geometries. The metasurface was designed to operate at $f=6 \mathrm{GHz}$ and each unit cell had a width and height of $\lambda_{0} / 10=5 \mathrm{~mm}$.

Each PCB unit cell was designed using Ansys HFSS, a fullwave electromagnetism solver, by illuminating the cell with a normally incident plane wave and using perfect electric

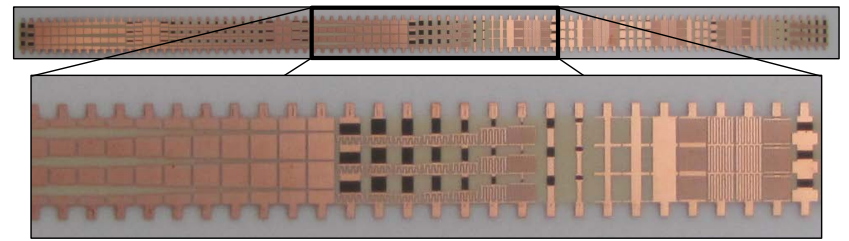

Fig. 1. The fabricated PCB design of the cylindrical impedance metasurface.

and magnetic conductor boundaries to have the unit cell appear to repeat infinitely in a sheet. Capacitive unit cells were designed using interdigital copper traces, where the capacitance was controlled by altering the overlap length of the digits or changing the number of digits. Inductive unit cells were designed with single line traces, where inductance was controlled by the width of the trace. Planar resistors were incorporated into each cell to introduce a series resistance with the reactive copper traces.

A $0.203 \mathrm{~mm}$ thick Rogers $4003 \mathrm{C}$ substrate with Ohmegaply resistor-conductor material was used to fabricate the complex impedance of each metasurface unit cell using the PCB design developed through simulation. The resitive portion of the surface impedance was implemented by forming planar resistors with the $25 \mathrm{ohm}$ per square Nickel Phosphorous layer of the Ohmegaply material. The reactive portion of the impedance was implemented through capacitive or inductive copper traces. The metasurface was fabricated with three identical vertical layers of the designed unit cells in order to ease the measurement process by increasing the height of the parallel plate waveguide. Fig. 1 shows an image of the fabricated PCB metasurface.

\section{Measurement Set-up and Results}

The design procedure made use of an infinite current line source as the enclosed radiating source and omitted $\hat{z}$ dependence, so image theory was used to achieve these properties by placing the source and metasurface within a parallel plate waveguide. We fabricated two copper circular ground planes to act as the parallel plate waveguide. Furthermore, we mounted an SMA-to-waveguide probe at the center to act as the current source and placed the metasurface in a cylindrical geometry at a distance of $r=1.5 \lambda=7.5 \mathrm{~cm}$. Microwave absorbing foam was placed at the circumference 


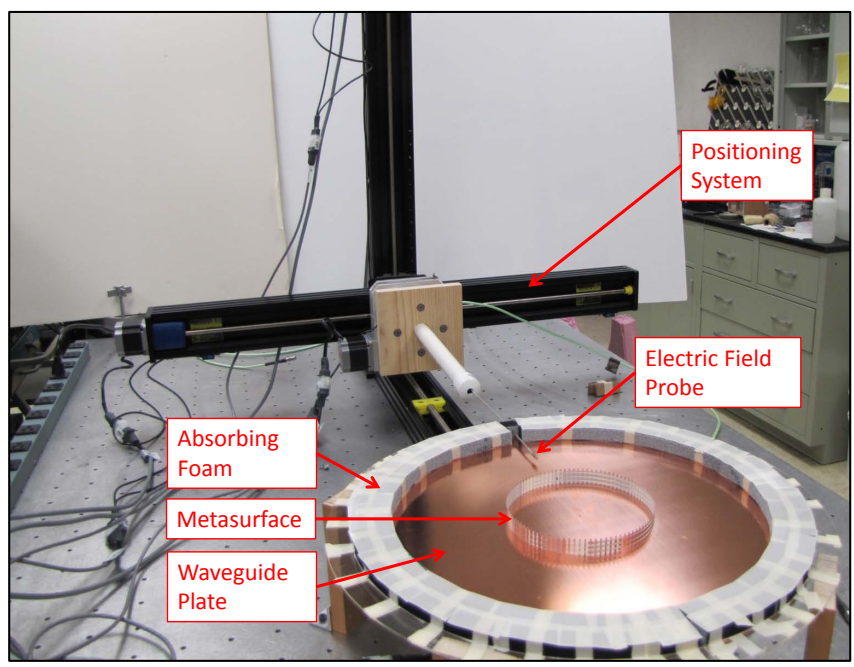

Fig. 2. The measurement set-up used to measure the transmission performance of the cylindrcal metasurface. The top plate of the parallel plate waveguide is removed to display the interior.

of the parallel-plate waveguide to absorb the energy radiated by the metasurface.

An electric field probe was used to measure the electric field intensity at various points within the waveguide. The probe was built using a semi-rigid SMA cable with a balun designed to cancel coupling from the exterior of the cable at $6 \mathrm{GHz}$.

The movement of the electric field probe in the waveguide was controlled using a stepper-motor positioning system with movement in multiple axis directions. The positioning system radially moved the electric field probe to control the radial distance between the probe and the cylindrical metasurface and rotated the entire waveguide to control the azimuthal angle of the probe in relation to the metasurface. The stepper motors used in the positioning system had a radial discrete step size of $6.35 \mu \mathrm{m}$ and an azimuthal discrete step size of 0.0125 degrees. This positioning system enables accurate cylindrical mapping of transmission measurements within the volume of the waveguide. Fig. 2 shows the PCB metasurface, waveguide (with the top plate removed), and positioning system used to take the measurements.

A vector network analyzer was connected to the SMA-towaveguide probe and the electric field probe to measure the two-port S-parameters within the waveguide volume. Since $S$-parameters are proportional to the voltage at the port, the $S_{21}$ measurement is an acceptable measure of the electric field intensity within the waveguide. Therefore, the azimuthal image pattern of the $S_{21}$ measurement can be compared to the desired azimuthal image pattern to verify the design.

We took an S-parameter measurement sweep at the image distance of $r=15 \mathrm{~cm}$ at one degree increments and compared the normalized $S_{21}$ measurements to the normalized simulated electric field values and theoretical pattern at the image distance. Fig. 3 plots these results, and we see that the transmission measurements are in very good agreement with

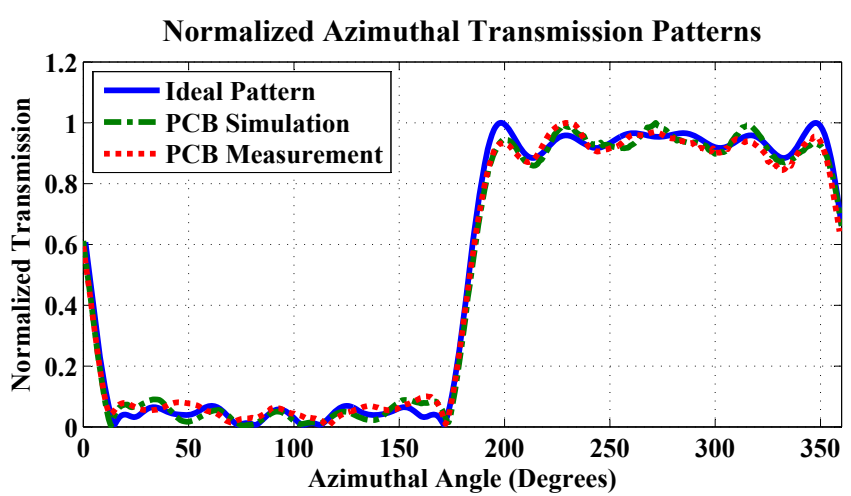

Fig. 3. Theoretical, simulation, and measurement results of the desired azimuthal image pattern.

the simulated and ideal patterns. Specifically, the roll-off of the azimuthal step function is nearly matched in steepness and the relative amplitudes in each region are nearly the same between measurement and simulation.

To provide a quantitative measure of performance between the normalized simulated and measured azimuthal image patterns, we calculated the normalized root-mean-square (RMS) error between the two patterns to be 0.021 . In comparing the measured normalized azimuthal transmission pattern of the metasurface to the ideal pattern, we calculated the normalized RMS to be 0.034 . As can bee seen from Fig. 3, the cylindrical metasurface is very effective at creating both a null region as well as realizing a target non-zero pattern shape.

We attribute the normalized RMS error to manufacturing tolerances and other physical non-idealities of the measurement set-up, such as the presence of very small gaps between the metasurface and the parallel plates of the waveguide.

\section{SUMMARY}

Overall, the agreement between the $S_{21}$ measurement and simulated image pattern validate the design method used to form a desired azimuthal radiation pattern using a cylindrical impedance metasurface. This method can be used to form azimuthal radiation patterns that are tailored for the surrounding environment. Introducing nulls to minimize the radiated power in specified directions and forming precise gain distributions as a function of azimuthal angle are two immediate examples, and can be done using existing antennas as sources.

\section{REFERENCES}

[1] C. Pfeiffer and A. Grbic, "Metamaterial huygens' surfaces: Tailoring wave fronts with reflectionless sheets," Phys. Rev. Lett., vol. 110, May 2013.

[2] F. Monticone, N. M. Estakhri, and A. Alù, "Full control of nanoscale optical transmission with a composite metascreen," Phys. Rev. Lett., vol. 110, May 2013.

[3] M. Selvanayagam and G. V. Eleftheriades, "Discontinuous electromagnetic fields using orthogonal electric and magnetic currents for wavefront manipulation," Opt. Express, vol. 21, no. 12, June 2013.

[4] B. O. Raeker and S. M. Rudolph, "Arbitrary transformation of antenna radiation using a cylindrical impedance metasurface," IEEE Antennas and Wireless Propagation Letters, 2015.

[5] A. Grbic, L. Jiang, and R. Merlin, "Near-field plates: Subdiffraction focusing with patterned surfaces," Science, vol. 320, no. 5875, 2008. 